\title{
العولمة: هل من رد إسلامي معاصر؟
}

* إبراهيم أبوربيع

الليبرالية الجديدة، كنظام عالمي هي في حقيقتها حرب جديدة لغزو مناطق جديدة فهي بالقطع لا تعني فاية الحرب العالمية الثالثة أو الحرب الباردة'، وأن العالم قد تغلب على الثنائية القطبية، واكتشف الاستقرار من جديد في ظل المنتصر. فعلى حين كان ثمة جانب مهزوم، وهو العسكر الاشتراكي، فإنه يصعب تحديد الجانب الفائز هل هو الولايات المتحدة؟ أم الاتحاد الأوروبي في المجال التجاري؟ ام اليابان؟ أم الثلاثة معا؟ فيفضل الحاسوب والأسواق المالية ووفقا لرغباتا تفرض الليبرالية الجديدة قوانينها ومبادئها على الكوكب. فلاتعدو العولمة أن تكون امتداداً شموليا لمنطقها إلى كل جوانب الحياة. الولايات المتحدة التي كانت حاكمة الاقتصاد سابقا، أصبحت الآن محكومة عن بعد بحركية القوة المالية الفائقة: التجارة الحرة. وقد استفاد هذا المنطق من خاصية النفاذ الناتحة عن تطور الاتصالات ليتولى أمر كل جوانب النشاط في المجال الاجتماعي، وكانت النتيجة حربا شاملة. 1

لقد كانت الثقافة في الخمسينات والستينات- وهي أحد مراحل تاريخ العالم الثالث والتي يأمل مؤيدو العولمة في تمميشها واغتيالها - تتشكل من نوعين من الثقافة: الثقافة الإمبريالية المهيمنة والثقافة الوطنية التحرية، ويرغب أولئك المتأثرون بأيديولوجية العولمة في ابتداع ضرب جديد من الثقافة: ثقافة الانفتاح

$$
\text { والتجدد أو الانسحاب والركود.2 }
$$

ويقال لنا اليوم إن العالم يتقارب وأن المسافات الشاسعة قد ألغتها الحاسبات الآلية والفاكس، وأن الأرض أصبحت الآن "قرية عالمية" أصبحنا فيها مترابطين على نحو غير مسبوق، ومع ذلك فثمة شعور

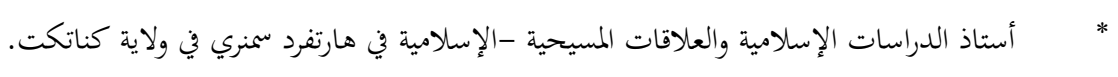

1 Sub-commandant Marcos, "La 4e guerr mondiale a commence", Le monde diplomatique (Augst 1997), P1. 
ينقض ذلك تماما؛ شعور بأن التنائي والانفصال يشكلان الحياة الحديثة. وإن كان ثمة شيء قد تقلص فهو الإحساس بالوجود الذي تعانيه الذات الحديثة. ويقرر علماء النفس أن مستويات الإحباط والقلق عالية، وأن شبكة الأصدقاء وأفراد الأسرة القريبين والعلاقات داخل المجتمع انكمشت بالنسبة لأغلب الناس إلى جزء صغير مما عايشته الأجيال السابقة. 3

إن المعرفة التي كانت متاحة وبلا مقابل ومن أجل صالح المجتمع، أصبحت الآن سرية ولصالح الكسب

المادي، وبعد أن كان أهل العلم يحرصون على استقلالهم العلمي هاهم الآن يخططون المناهج العلمية وفقا لما يتم الاتفاق عليه مع مؤسسات الأعمال الممولة وأصبح الأساتذة الذين كانوا يدرسون مقيدين في قوائم رواتب الشركات التي يقومون لها، في مختبرات الجامعات، بأبحاث يمكن تسويق ما تسفر عنه، بينما تدفع الجامعات رسوما عخفضة لإحلال الأساتذة المساعدين، أما رؤساء الجامعات الذين كانوا القادة المفكرين لتلك

$$
\text { المؤسسات فقد أصبحوا الآن يتمثلون بالتجار المتجولين (جون هاريس).4 }
$$

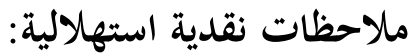

يستحيل تقريبا تقديم تعريف بسيط لمصطلح "العولمة" نظرا لأنه يحمل عددا من الدلالات المتداخلة في

المجالات الاقتصادية والاجتماعية والسياسية والايديولوجية والفكرية. ويعنيني في هذه المقالة إثارة عدد من الأسئلة الحساسة حول هذه الدلالات خصوصا في علاقتها بالعالم الإسلامي المعاصر، وتقديم بعض الملاحظات النقدية حول حالة الفكر الإسلامي المعاصر، واقتراح طرق مواجهة التحولات المعرفية والأخلاقية والعلمية التي أحدثتها العولمة مؤخرا. وبداية أود القول إن مؤيدي العولمة أساسا لم يأخذوا المنظور الإسلامي

3 Charles Spretnac, The resurgence of the real: Body, nature, and place in a Hypermodern world (New York; Addison- Wesley, 1997), 11.

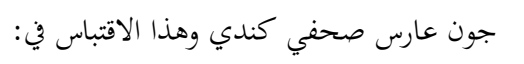

Maude Barlow and Geather- Jane Robertson, "Homogenization of Education", in "A case against the Global Economy: And for a true toward the local, Editied by jerry mander and Edward Goldsmith (San Fransisco: Sierra Club Books, 1996), 62-63. 
في الاقتصاد والمجتمع بجدية، لأن هذه الظاهرة لم تحظ بتقدير ونقد إسلامي بشكل منتظم. كانت هناك حركات مفاجئة ضد الحداثة الغربية كان أبرزها في إيران والسودان ومصر، إلا أن ردود فعل المثقفين المسلمين على المشاكل الناجمة عن العولمة لم تتحقق برغم أنه قد حان وقتها من زمن بعيد.

وبغض النظر عن الاتجاهات المعرفية المعقدة المستبطنة في الفكر الإسلامي المعاصر، فإن هذا الفكر لم يكقق كل إمكاناته الفكرية، لنه أخفق في معالجة أكثر القضايا أهمية في زماننا؛ وهي غيبة النقد والفهم الإسلامي للقومية الحديثة والديمقراطية والحداثة والدولة القومية بل وحتى للاستعمار والاستعمار الجديد. فباستثناء دراسات وانطباعات فردية قليلة لم يقدم الفكر الإسلامي المعاصر منظورا شاملا، ناهيك عن أن يكون مقنعا، للعديد من القضايا والمسائل التي تكتنف العالم الإسلامي المعاصر. وهذه الثغرة أكثر وضوحا في موضوعي الحداثة والعولمة.

ولإلقاء الضوء على طبيعة الفكر الإسلامي الحديث ينبغي التمييز بين الفكر الإسلامي والفكر العربي

أو الباكستاني؛ فقد يعني المرء بالفكر العربي الحديث الإنتاج الفكري للمفكرين الدينيين والعلمانيين العرب في العوام المائة للأخيرة، بعبارة أخرى، يجب عدم تسوية الفكر العربي بالفكر الإسلامي؛ لأن الفكر العربي يشمل كل نزعات وابتحات وأنماط الفكر التي تعكس المسائل والقضايا المحيرة التي تشغل الفكر العربي الحديث. 5 ومن ناحية أخرى فإن الفكر الإسلامي أساسا لاهوتيا وله كذلك إطار مرجعي علمي يحدده الوضع المحوري للقرآن الكريم في الحياة والفكر الإسلاميين.

تستدعي الملاحظات السابقة التساؤل حول طبيعة الحضور الإسلامي في الغرب بصورة عامة، فمن

المغالطة القول إن الحضور الإسلامي في الغرب ليس مهما للعالم الإسلامي، لأن هذا الادعاء ليس له ما يؤكده، فمع موجة التحديث هجرة المثقفين المسلمين والمهندسين والأطباء والمهنيين إلى الغرب منذ بداية القرن العشرين، أصبح من المهم إثارة الأسئلة حول طبيعة واتحاهات الإسهامات الفكرية الإسلامية في

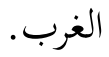

$$
\text { انظر الفصل الأول من: }
$$

Ibrahim M. Abu-Rabi, intellectual origins of Islamic resurgence in the modern Arab world (Albany State University of New York press, 1996). 
ولم ينتج المسلمون في الغرب- إجمالا- مفكرين من بينهم يستطيعون مساعدة الوجود الإسلامي في احتكاكه اليومي بالحداثة والعولمة، ويمكن استثناء عدد قليل من الأكادميين الذين يتبنون المنظور الإسلامي. وإن مثل التجمعات الإسلامينة في استيعاب المشكلات المركزية المحيطة كمم في بيئة غير إسلامية - وإن كانت متسايحة دينيا- يعكس المعاناة النفسية والاجتماعية للمسلمين في وسط غربي. والمجتمع الإسلامي في مثل هذه الحالة هو في وضع مثالي من حيث قدرته على إنعام النظر من الباطن كما- يقولون- في طبيعة العولمة، وإرشاد العالم الإسلامي لفهم المخاطر الناشئة عن الليبرالية الحديثة وقوى السوق الجديدة. ومن المستحيل التهرب من الجزم بان المجتمع الإسلامي في الغرب مضطر لتطبيق قيمه على حقائق العالم الجديدة التي لا تتفق وأية رؤية توحيدية للعالم في حين تتواءم ومعايير العالم الاستهلاكية والتنافسية.

ونتيجة للعوامل السابقة فإن ثمة هاجسا يتعلق بقضية التراث وكيفية الحفاظ عليه في عالم سريع التغير وأحيانا لا يرحم. ولا يعني هذا أن قضية التراث لم تعد مهمة، إلا انه يبدو لي انه حان وقت بتحاوز الصيغات المفاهيمية لمفكري القرن التاسع عشر المسلمين من أمثال محمد عبده وجمال الدين الأفغاني والسيد أحمد خان، وذلك من خلال ابتكار أسلوب إسلامي جديد للتفكير، ويستجيب بشكل مبدع للقواعد الصارمة للفكر الفلسفي والأخلاقي، فلا يمكن لأي فكر أن يسبر غور إشكاليات العولمة ما لم يستوعب بصورة كاملة الاتحاهات الحديثة في النظرية النقدية والفكر الاجتماعي والاقتصادي المعاصر وعلاقاتها بالفكر الديني في العالم الإسلامي والغربي، ورد الفعل الأخلاقي الكيد الذي يجب أن يقدمه الفكر الإسلامي المعاصر لتأكيد حيويته وضرورته. مستعينا بالأدوات النقطية وبمنطق الوحي لتقديم إجابات شافية لمشاكل العالم الإسلامي المعاصر.

\section{ثلاث مقدمات منطقية وعديد من البراهين:}

يجب على الفكر الإسلامي الحديث لكي يستعيد حيويته أن يعيد تفسير المبادئ المعيارية والدينية الأساسية للإسلام بطريقة تعارض الطبيعة الشمولية للأنساق السياسية والتربوية الحاكمة في العالم الإسلامي المعاصر، والدعم الذي تلقته مع سيطرة العولمة على السوق العالمي والنفس الإنسانية. فإذا ما قبل المرء بان 
المساواة بين البشر هي صلب الرؤية الإسلامية، توجب عليه أن يخلص إلى أنه يلزم لاتباع المثاليات الإسلامية معارضة أشكال القهر السياسي والاقتصادي والاجتماعي والفكر التي تسيطر حاليا على العالم الإسلامي وبعبارة أخرى يجب علينا تقديم رؤية إسلامية للعالم ذات طبيعة تحرية، وأن تكون ذات معنى للإنسان العادي

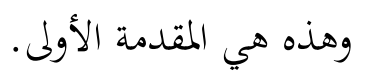

أما المقدمة الرئيسة الثانية فهي تاريخية إلى حد ما، وتتصل بالأساس بالتغيرات الاجتماعية الاقتصادية الهائلة الجارية في الغرب الرأسمالي الحديث وتأثيراتها السياسية والفكرية على العالم والفكر الإسلامي المعاصر عموما. ومن السذاجة افتراض أن الفكر الإسلامي الحديث يتبع آليات داخلية معينة ليس لديها ما تقدمه إزاء التحولات المعقدة في الفكر الغربي الحديث، أو أن الفكر الإسلامي الحديث يرفض الاستعارة من مصادر خارجية. إن إدراك طبيعة التحولات الاقتصادية والسياسية والفلسفية في العالم الغربي الحديث يعني التعالم بجدية مع بجمل تاريخ الفكر الغربي، من الماركسية والماركسية الجديدة إلى الرأسمالية ثم العولمة وهي آخر بحليات ذلك الفكر، وقد يحاول بعضهم بأن تاريخ الغرب، وبالأخص الأمريكي والأوروبي، شهد تحولات رئيسية في رؤيته للعالم وآخرها العولمة، في حين ل تزال الإشكاليات النابحة عون الحداثة تعجز الفكر

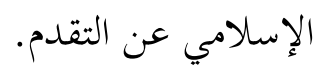

تبدو العولمة لأغلب المفكرين في العالم الإسلامي، بدءا من الراديكاليين (المنادين بالتغير الجذري) إلى المحافظين، ظاهرة محتومة. ومن الصحيح بمعنى من المعاني، أن مصير العالم الإسلامي متداخل أشد التداخل مع الرأسمالية الغربية المنتصرة بسبب الطفرات التاريخية والسياسية والفكرية في الغرب الحديث، ومع ذلك تبقى أسئلة عديدة تنتظر الإجابة: فماذا تعني العولمة في سياق عالم مابعد الاتحاد السوفيتي وما بعد الحرب الباردة

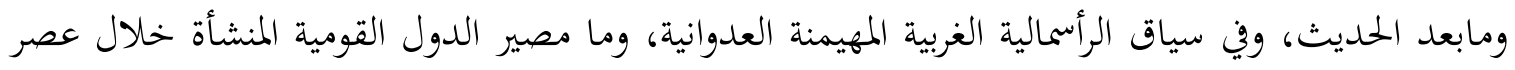
الحرب الباردة؟ كيف تغير المجتمع المدني في العالم الإسلامي في العقد الماضي؟ وهل اهيار الاقتصاد والمجتمع الأندونيسي مؤخراً نتيجة مباشرة لتجاوزات العولة؟ وما دور المفكرين المسلمين في عصر التحول الحاد هذا؟ كيف يتأتى الحفاظ على الملامح الأساسية للهوية الإسلامية في السياق السالف، خاصة إذا كانت هذه 
الملامح قد تم الإعراب عنها في مرحلة ماقبل العوملة؟6 وإذا ترجمت المحافظة على أها آلية دفاعية، فما هي الأدوات المفاهيمية التي يجب ابتداعها لإعادة حيوية الفكر الإسلامي الحديث في تعامله مع العولمج؟ وأخيرا لماكانت صناعة القبول في المجتمع المعاصر تقوم على تأثير الأفكار، ويجري تعليم الأفكار الرأسمالية الأساسية في المدارس الخاصة والجامعات في أنحاء العالم الإسلامي فأين تكمن المصلحة العامة للشعب؟

المقدمة الثالثة مشتقة من السؤال الأخير. فالعالم الإسلامي يمر بعملية درامية من التغير في نظمه التعليمية، والتعليم الأفضل تعليم خاص للمحافظة على أبناء النخبة وهي النخبة نفسها التي شنت ثورة فكرية صامتة عظيمة الدهاء ضد الجماهير في العقود القريبة. ويظهر انتشار المؤسسات التعليمية الخاصة ومراكز التعليم عن بعد في تركيا ومصر والأردن وماليزيا والسعودية والمغرب، بشكل لا تخطئه العين مما يؤكد أن التعليم

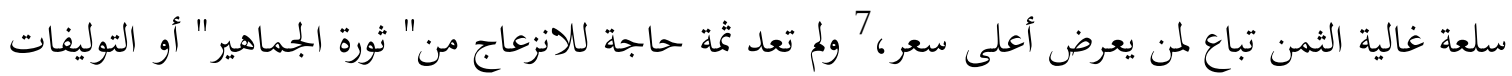

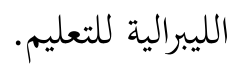

وبينما استمرت قدما خصخصة التعليم وإكسابه طابعا نخبويا وغربنته وأمركته بدرجة لها دلالتها في العديد من أقطار العالم الإسلامي كان عامة الناس قد سلبوا كبرياءهم التقليدي، وعمّ وعي جديد على أساس من الفارق الطبقي التعليمي والتمايز الاجتماعي الاقتصادي، ويصح تطبيق الملاحظة التالية عن رجال القبائل في شمال شرق الهند على العالم الإسلامي:

لايمكن إنكار قيمة التعلم الحقيقي أي توسيع وإناء المعرفة، لكن التعليم أصبح اليوم في العالم الثالث أمرا مختلفا تماما، فهو يعزل الأطفال عن ثقافتهم وعن الطبيعة، ويدربمم عوضا عن ذلك، على أن يصبحوا متخصصين بشكل دقيق في بيئة حضرية مغربنة، وتظهر هذه العملية بشكل صارخ على وجه الخصوص في

$$
\text { لقيت أندونيسيا مؤخرًا تغطية إعلامية كبيرة في الغرب، راجع المقالات التالية: }
$$

V.S. Napul. "Indonesia the man of moment, "The New York Review of Books 911 June 1998), 40-45; Noam Chomsky," L'Indonesia atout maitre du jeu American, le monde diplomatique (june 1998).

انظر

Gerard De Selys, Tableau noir, appel a la resistance contre la privitasisation de l'ensiegmwnt 9Bruxelles: EPO, 1998). 


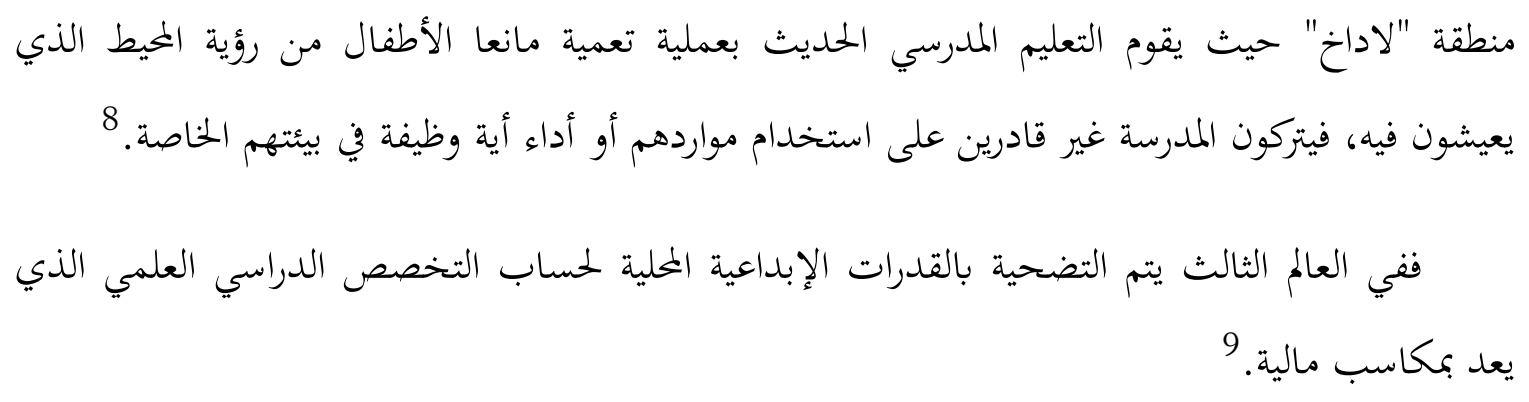

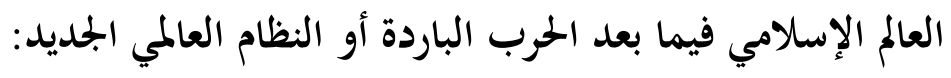

\begin{abstract}
العولمة ليس ظاهرة جديدة، فقد ظهرت مع انتصار رأسمالية "دعه يعمل" في عصر ما بعد الثورة
الصناعية والتوسع الاستعماري الأوروبي، بعبارة أخرى أحدثت الإمبريالية الأوروبية المنتصرة تحولات عميقة

سياسية واقتصادية وثقافية ودينية في ما يدعى العالم الثالث وأدت هذه التحولات إلى تفاعل دائم بين الشمال والجنوب، 10 وكانت السيطرة الاستعمارية ذات حدين: إذ أدت إلى تحديث بعض المؤسسات والقطاعات المهمة في المستعمرات كالجيش وقوات البوليس والنظام التعليمي، ولكنها خلقت أيضا اختلالا في التوازن الاجتماعي والاقتصادي، من قبيل الفجوات الأساسية بين الريف والحضر التي أدت إلى هجرة ريفية إلى المدينة، وإلى نخبة فكرية مزدوجة اللغة وتتصف بالقلق. 11
\end{abstract}

8 Helena Norberg, "The Pressure to modernize and Globalize".

$$
9
$$

Theodore Roszac, The Cult of information: The Folklore of computers and the True Art of Thinking (New York: Pantheon Books, 1986); Vandana Shiva, Mono-Culturees of Mind: Biodiversity, Biotechnology and Third World (Penang Malaisia: Third World Network, 1993).

$$
\text { في حالة الهند على سبيل المثال انظر: }
$$

R. Mukhrjee, The raise and Fall of The East India Company (Berlin); Veb Duesscher Verlag der Wissenschaften, 1958.

11 Edward Sjill, The Intellectuals and the Powers and Other Essays (Chicago: The University of Chicago Press, 1972).

وبالأخص الفصل الثالث Intellectuals in Underdeveloped Cpuintries 
لقد كانت استجابة الدولة القومية لتغلغل رأسمالية الطبقة البرجوازية تؤكد على الاستقلاب الوطني الاقتصادي والثقافي، وتسعى فنشاء بنى وأسس جديدة للدولة الجديدة لتجاوز الاعتماد التقليدي على الاستعمار ومنح وجود الاتحاد السوفيتي والحرب الباردة الدول القومية الجلديدة حيزا للمناورة، ومع ذلك فقد أخلَّ حدثان رئيسيان بالتوازن في السبعينات والثمانينات لصالح الغرب الرأسمالي: الأول هو غزو الرأسمالية الأمريكية للسوق الصينية الضخم، 12 واهيار النظام السوفيتي وما تلاه من انتهاء الحرب الباردة، التي كانت علامة بارزة على انتصار الغرب الرأسمالي على الشرق الاشتراكي، 13 وترك هذان الحدثان الرئيسيان المنقطعا النظير تاريخيا، الدول القومية فرائس سهلة لتحديات وأخطار النظام العالمي الجديد وحقائقه الاقتصادية والجغرافية السياسية.

يدل هذا التغير مرة أخرى على أهمية القرارات الاقتصادية في تحديد ابتحاه السياسة العالمية في المستقبل.

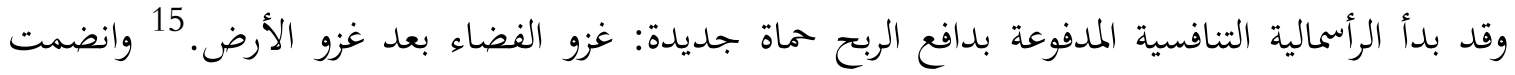
إليها الشركات متعددة الجنسية التي ترى مستقبل الرأسمالية في غزو فضاء جديد وأقاليم لا مادية جديدة، وتسعى -للنجاح في ذلك- لإنشاء مناخ علمي يسمح بالتنافسية بدون اليد المتحكمة للدولة. وهذا الاتجاه من الرأسمالية لعدوانية اندفع جنوني، مسعور بسبب غياب نسق سياسي واقتصادي دولي تعددي وقد لاحظ احد المؤلفين: "أنه ظل نسق الليبرالية الجحديدة تتولى الشركات الخاصة، بالتنسيق مع الدول والهيئات الدولية المسؤولة عن مساعدقا، في الترويج لمجتمع والرفاه والإنساني. وتنتج هذه الشركات السلع، وبشكل متزايد

12 H. Kissinger, Yeans of upheaval (Boston, Little, Brown and Company, 1982), and the White House years (Bosten: Little, Brown and Company, 1979).

$$
\text { حول الآثار الداخلية للحرب الباردة على الولايات المتحدة انظر: }
$$

James T. Paterson, Grand Expectations: The United States, 1945-1974 (New York; Oxford in University Press, 1972

14

J. Decornyon, capital Priove, Development du Sud et Solidarite Mondiale: Les Multinationales, Omnipresentes et... impuissantes " le monde Diplimatque (November 1988), 8-9.

15 B. Karlin, "Space New Frontier for US Enterpreneurs" International Heradl Tribune 914 September 1988). 
الخدمات، ويتجاهل أكثرها فعالية الحدود، والربح هو علة وجودها ومركها الوحيد والحقيقة أن فجوة كبيرة تفصل مابين الأهداف وبين القدرة على الوصول إليها، وحتى لو لم نضع في خلفية تفكيرنا هذه الشركات التي أخفقت في الغرب، فإنه يجب التأكيد على أنه لا أمل للجنوب. 16

ولقد كان لإنشاء مناخ معولم سهل الانقياد لنمط جديد من الربح الدول آثار سلبية على الاقتصاديات المحلية الداخلية، بمعنى أنه جاء بمجموعة جديدة من قواعد الرشادة والقيم لا تأتلف مع تقاليد وثقافات وتاريخ سكان البلاد الأصليين والدول القومية، إذ أن منطق الرأسمالية ليس الحفاظ أو إنماء أنساق اجتماعية اقتصادية سليمة بل جني الربح بأسرع ما يمكن. 17

تستدعي الفكرة الأخيرة التساؤل حول العلاقة الجديدة بين الشركات متعددة الجنسية والدولة القومية، من حيث دور الدولة في المجتمع، والتغيرات العميقة في علاقات القوى، ليس بين الشمال والجنوب فحسب، بل بين دول الجنوب أيضا، وطبيعة المجتمع المدني، وتضمينات هذا للديمقراطية في العالم الثالث. 18

تتحدى العولمة الدولة القومية لتفتح فضاءها وحدودها لنمط جديد من التنافس المتحرر من أية سيطرة، 19 ويتوقع من النخبة السياسية في الدولة القومية أن تعاون بشكل كامل مع المشروعات الاقتصادية، وغالب ما تكون مراكمة رأسمال وطني مستحيلة، لأن العديد من دول العالم الثالث مثقل بدون كبيرة للمؤسسات المالية الدولية، وهنا تتغير قواعد اللعبة، فالتنمية الوطنية والنمو في العالم الثالث مقيدان بتراكم رأس المال على نطاق دولي.

16 Decornoyn, "Capital Prive" 9.

$$
17
$$

18 Z. Laidi nd et al., L'order mondial relache (Paris: Presses de la Fondation natioales des sciences 1994).

انظر:

19

S. Strange, The retreat of the State: The Diffusion of in the World Economy (Cambridge: Cambridge.

University Press, 1998). 
كانت العولمة في الخمسينات وفقا لرؤية سمير أمين محكومة إلى حد ما بثلاثة عوامل دولية: تدخل الدولة الرأسمالية في عملية تراكم رأس المال، والمشروع السوفيتي لإقامة اقتصاد اشتراكي، ومشروع باندونج من أجل عالم غير منحاز تحت رعاية سوكارنو وغرو وناصر. 20 ومع انتشار الخصخصة في بلاد هؤلاء لقادة الراحلين، وصل المشروع الوطني الاشتراكي المتعلق بكل من الاكتفاء الذاتي والتمكن للفقراء إلى حد التوقف المميت، في العالم العربي على سبيل المثال، اقتطعت الخليج قسرا من بقية العالم العربي، وكثف المركز الغربي هيمنته اقتصاديا وعسكريا في أعقاب الهزيمة العسكرية للعراق في حرب الخليج الثانية، ويعني وذلك وفقا لرؤية سمير أمين، أن دول الخليج "أصبحت الان محميات مجردة من أية حرية للمناورة اقتصاديا أو سياسيا" 21 أو بتعبير إيمانويل ولرشيتن كسب العالم الثالث المعركة السياسية في الخمسينات والستينات حيث تم التخلص من الاستعمار في كل مكان تقريبا، وحان وقت الحنطوة الثانية وهي التنمية الوطنية، ولم تنجز هذه الحخطوة في أغلب المواطن، 22 ففي عصر اللبرلة والاندماج لا تسمح العولمة للاقتصادات المحلية بأن تحيا مستقلة بنفسها. 23 إن إخفاق الاقتصادات الوطنية في أقطار مثل اندونيسيا والهند ومصر والجزائر دليل انتصار التكنولوجيا العقلانية في الغرب المتقدم، ومع ذلك، فكما يذهب بعض الاقتصاديين لايمكن أن يتحدد انتشار اللبرلة المالية أو العولمة التكنولوجية بعامل التكنولوجيا وحده؛ فالسياسة لها دور كبير في انتشار التكنولوجيا العقلانية

\section{: $\quad 20$}

Samir Amin, Les defies de la mondialisation (Paris: L'harmattan /Forun du tiers-monde, 1996).

$$
22 \text { انظر ، } 21 \text { انظر مواجهة أزمة عصرنا مرجع سابق ص } 133 .
$$

Immanuel Wallerstein, After Liberalism (New York: The New Press, 1995), 15.

Daniel T. Griswol, "Blessings and Burdens of Globalization, "The Worl and 1 (April 1998): 30-35. 


$$
\begin{aligned}
& \text { والعولمة، والرغبة السياسية للمركز وهي أساسا إخضاع كل الدول القومية - مع استثناء محتمل لإسرائيل - } \\
& \text { لمقتضيات السوق الرأسمالي.24 } \\
& \text { يتصور مؤيدو العولمة نوعا من "|قتصاد القرية العالمية" ويسهل انتشار التحديث والترشيد التكنولوجي } \\
& \text { في العالم، ويزعمون أن العولمة أدت إلى إدماج المجتمعات التي جرى إفقارها وتميشها حتى الآن في السوق } \\
& \text { العالمي. ولم تؤد العولمة في رؤيتهم إلى خلق ملايين الوظائف العديدة وتحسين الظروف الاقتصادية والاجتماعية } \\
& \text { للفقراء فحسب، بل وأدت أيضا لانفتاح الآفاق الثقافية والفكرية للأمم الفقيرة، } 25 \text { وحتى لو بل المرء مثل هذه } \\
& \text { الرؤى،ة التي أصبحت عملية لا يمكن بتنبها في العديد من أقطار العالم الثالث، فإن عليه أن يغمض عينيه } \\
& \text { عن العمليات الأخرى المصاحبة لما والتي لا تقل عنها دلالتها، وهي أن النظم المتعولمة أشد رسوخا مما كانت } \\
& \text { عليه النظم الشمولية في الأعوام الماضية، } 26 \text { فقد خلق قمع الدول القومية طرقا جديدة تقمع بها الدولة القومية } \\
& \text {-القموعة- المجتمع المدني، وقوض هذا القمع التماسك الاجتماعي في العالم الثالث، وقلص الحيز الديمقراطي } \\
& \text { في المجتمع، واختنق المجتمع المدني نتيجة للتحولات العديدة في حدود دوائر القوة في المجتمع. وأصبحت حرية }
\end{aligned}
$$

24 P. Vairoch and E. Helleiner, States Against Markets: The Limis of Globalization (London:

Routledge, 1996).

25 Peter Martin "la mondialisation est - elle Invitable? Une obligation morale" La Monde Diplomatique

(June 1997).

26 Ignacio Ramonet, "Regimes globalitaires", Le Monde Diplomatique (January 1997), I Accoriding to ramonet, "On appelait regimes totalitaires" ces regimes a parti uniqe qui n'admettaient aucune opposition organize, qui subordannaient les droits de la personne a la rason d'Etat, et dans lesquels le pouvoir politique dirigeait souverainement la totalite des activates de la societe domineer. A ces systemes succeed, en cette fin de siecle, un autre, un autre type de totalitarisnem celui regimes globalitaries'. Reposant surles dogmes de la globalisation et de la pensee unique, ils n'admettent aucume autre politique economiquem, subordonnet les droits sociaux du citoyen a la raison competitive, et abadnfonnent aux marches financiers la directyion totale des activities de la societe domineer" ibid. 
التعبير سلعة نادرة. 27 وكما لاحظ مؤلف فطن فغنه "بينما كانت الول المصنعة تظهر تدريجيا، كان النظام العالمي الجديد لاذي فرضه الاقتصاد المعولم يعني للجميع سباقا نحو القاع، فتم خفض الوظائف باسم التنافس العالمي، واعتلت الشركات عابرة القوكيات مستوى من القوة فوق أية حكومة، وحتى في دولة مثل الهند حيث يرلف عشرة في لالمائة من أصل تسعمائة وتمانين مليونا من السكان طبقة وسطى جديدة، تستفيد من الحضور العالمي للشركات عابرة القوميات فإن التسعين في المائة الأخرى ليسوا مدرجين في خطة المستقبل المبهر، ويشكل العشرة في المائة، بدخولمم المتاحة، سوقا واسعة بدرجة تكفي لإسالة لعاب اللاعبين العالميين، وأصبحت الهند بذلك قصة نجاح عظيمة. 28

استمر الحيز الديمقراطي في الدولة القومية الجديدة في الثمانينات والتسعينات عرضة لتحديات في الصميم، فربما يتوقع المرءء أن يقوم المركز قدم حامل لواء الديمقراطية لأمد طويل بتعزيز ديمقراطية حقيقية في الجنوب، إلا أن هذا بعيد عن الحقيقة، فقد تشكلت في ظل العولمة علاقة جديدة بين النخبة السياسية والقوى الاقتصادية، وبالخص الشركات متعددة الجنسية، 29 ويعني هذا في العالم الإسلامي أن الدولة القبلية شبه الدستورية، التي سيطرت عليها العائلة أو العشير نفسها كسبت قوة قمعية إضافية، وعانى المجتمع المدني من الخسارة الإضافية للحرية، وسلب العمال والمأة من كل فاعلية.

إن تغلغل الشركات متعددة الجنسية في اقتصاديات العالم الثالث -بعيدا عن أن يقضي على الفقر أو يخفف بؤس فقراء الريف- أدى إلى ثلاث ظواهر متضافرة: زيادة عدد الناس المعطلين والفقراء، وتركيز الثروة في أيدي النخبة السياسية، وزيادة قمع الدولة30 واندونيسيا مثار بارز لذلك، فمع قدوم التقنيات الحديثة في

27 Paul Hirst and Graham Thompson, Globalization in Question: The international Economy and the Possibilities of Government (Cambridge: Polity Press, 1996).

28 Spretnal, Resurgence of the Real, 34.

29 C.de Brie, "Le couple Etat-nation en instance de divorce" Le Monde Diplomatique ( May 1989).

30

Nancy Bridsall, "Life is Unfair: Inequlity in the World" Foreign Policy (Summer 1998): 95113. 
زي العولمة، أعيد التأكيد على الرسالة الغربية القديمة أو الرسالة الحضاري في ظل الآلهة الجديدة/ القديمة لكل

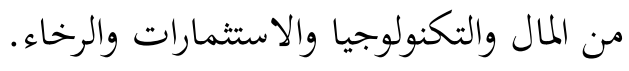

لقد تحولت صورة نخبة الفكر في العالم الثالث جذريا في عصر العولمة، ففي عصر الاستعمار قامت نخبة الفكر المحلية بدور ريادي في كل من الاستقلال السياسي والثقافي على الرغم من أها كانت وفق رؤية بندكت اندرسون "نخبة فكرية منعزلة مزدوجة اللغة واستحوذ عليها القلق". 31

إلى أها عكست هوم ومعانات شعبها، وحاربت من أجل الاستقلال عن سادقمم المستعمرين ومدارسهم التي درسوا فيها. أما نخبة الفكر في مناخ "عولمة العالم الثالث" فلا تشاركها هذا القلق، فالعلماء والفنيون والمهندسون والباحثون يجمعهم الانشغال بالعمال والاستثمارات، ولكنهم يعبرون عن أنفسهم بأسلوب جديد من اللغة الإنجليزية وهو أسلوب مدرسة لندن للاقتصاد أو مدرسة هارفارد للعمال، وبتعبير "بورديو" يميل هؤلاء الفنيون الجدد إلى تفضيل الربح الاقتصادي على حساب اختلال الأوضاع الفكرية والاجتماعية في ججمعات العالم الثالث، ويدعم الفنيون ما يسميه بورديو "العنف الهيكلي" في هذه المجتمعات أي زيادة عدد المعطلين والمهمشين في المجتمع. 32 تنهض الغة الإنجليزية، وتحديدأ الإنجليزية الأمريكية، بدور رائد فغي عصر العولمة فلايمكن أن يكن الفني ناجحا دون إتقان أسرار إنجليزية الأعمال الأمريكية، وإذ صح أن الإنجيزية الأمريكية تطورت فغي رحم الإنجليزية البريطانية خلال القرون السابع والثامن والتاسع عشر، فمن الصحيح بالدرجة نفسها أن الإنجليزية الأمريكية كانت تستجيب خلال هذه الفترة بشكل خلاق للظروف الاجتماعية والاقتصادية للعالم الجديد، فقد طور الأمريكية في معركتهم ضد السيطرة البريطانية مرعية لغوية لا تشترك في القواعد القديمة مع إنجليزية

31 Benedict Anderson Imagined Communities: Reflections on the Origin and Spread of Nationalism (London: Routledge, 1991) 140.

32 Pierre Bourdieu, "L'essence du neoliberalism, "le Mionde Diplomatique (March 1998),3. 


$$
\begin{aligned}
& \text { الملكة، وقد عزز "نوح وبستر" اللغوي الأمريكي البارز في القرن التاسع عشر الفهم بأنه "من المستحيل إيقاف } \\
& \text { تقدم اللغة فهي أشبه بمحتوى المسيسبي الذي قد تدق أحيانا ملاحظة حركته. } 33 \\
& \text { لقد أخفقت النخبة السياسية الأمريكية خلال الحرب الباردة 1945-1989م في نشر اللغة } \\
& \text { الإنجليزية في مواجهة أعدائها، أساسا، لأن الإنجليزية الأكاديمية كانت حكرا على المفكرين اليساريين الذين } \\
& \text { تخلوا عن وصاية الكتب العظيمة والقيم التقليدية، وأفسدوا النظام الاجتماعي } 34 \text { ومع ذلك، فقد اتخذت } \\
& \text { إنجليزية الأعمال مع اهيار الاتحاد السوفيتي وهاية الحرب الباردة وضعا هجوميا، وتبني التكنوقراط الجلدد في } \\
& \text { الشمال والجنوب نمطا رسميا من الإنجليزية أكثر مساعدة لعالم المال والأعمال منه لعالم الإنسانيات. } \\
& \text { إن زيادة البؤس والفقر الاقتصادي ليست حكرا على العالم الثالث وحده، } 35 \text { فاليوم تنتشر هذه الظاهرة } \\
& \text { في لاقلب كما في فرنسا وإنجلترا والولايا المتحدة، فالتحلل الحضري باد في مدن المراكز الرئيسة، وهو في معناه } \\
& \text { العميق عرض لنقص الرفاه الاجتماعي والتعليمية بين الفقراء، ويدل تعاطي المخدرات وانتشار الجريمة المحلية } \\
& \text { على أن نسبة لها دلالتها من المجتمع في المركز المتقدم تم تمميشها، بعبارة أخرى، كسبت الرأسمالية في سعيها } \\
& \text { لمراكمة رأس المال، المزيد من الربح ولكن على حساب نشر التدهور في القطاعات الضعيفة من المجتمع } \\
& \text { داخليا وفيما وراء البحار، وكما يوضح أحد المؤلفين "تولد الرأسمالية الرخاء والفقر دعا في الوقت نفسه". } 36
\end{aligned}
$$

33 H.L. MencKen: The American English. An Inquiry into the Development of English in the United States. (New York: Alfred Knof, 1955): 25.

34 Richard Ohman "English and the cold War," in the Cold War and the University: Toward an Intellectual History of the Cold War Years, edited by Noam Chomsky et al., (New York: The New Press, 1997), 73.

35 E.J. Perkins, The World Economy in the Twentieth Century (Cambridge: Schenkman, 1983).

36 M.Beaud, "Janmais Tant de Richesses, Jamais tant de Misere...Sur les causes de la Pauvrete des nations et des himmes dans le monde contemporain." Le Monde Diplomatique (November 1997), 11. 
وكما سلف لم يظهر النظام العالمي الجديد م فراغ، فقد أصبح العالم الثالث مع سقوط النظام السوفيتي

في أوائل التسعينات، بعد عقود من النضال الحاد، ضحية سهلة للهيمنة الأمريكية، 37 وبغض النظر عما إذا كان العراق هدف بغزو الكويت سنة 1990 تحدى السيطرة الغربية، عموما، وسيطرة الولايات المتحدة خصوصا، فقد بث تجميع الحشود الغربية في الخليج تحت لواء الأمم المتحدة موجات صادمة عبر العالم الإسلامي، وحافظت- إن لم تكن عززت- الهزيمة العسكرية للعراق على مصالح الغرب القومية التي قوت -في مفارقة تاريخية واضحة- نظم الخليج السياسية التسلطية، ومنعت أية فرصة حقيقية لتحقيق الديمقراطية لأعوام

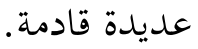

وبالإضافة إلى إبراز التحولات الرئيسية في الحلاف العالمية، وشهود ظهور الولايات المتحدة كقوة عظمى وحيدة، أثار هذان الحدثان، مرة أخرى وربما بشكل أكثر حدة قضية الهيمنة الثقافية الغربية، وخصوصا الأمريكية، وآثارها على ثقافات العالم الثالث. أما محدودية التأثير الأمريكية على العالم الإسلامي، في عصر ما بعد الحرب الباردة، في نطاق المسائل الاقتصادية والسياسية، فأمر أبعد ما يكو ن عن الصحة؛ لن هذا التأثير فكري ومفاهيمي في المقام الأول ولأن النخب الإسلامية الحاكمة تبنت الأفكار الرأسمالي

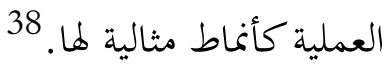

Charles Wiliam Maynes, "the Perils of an Imperial America," Foreign policy (Summer 1998): 36-49.

38 The Grand Crossboard American Primacy and Its Geostrategic Imperative ': New York " Basic

Books (1997), especially Chapter Two.

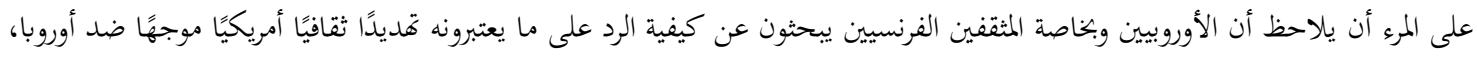

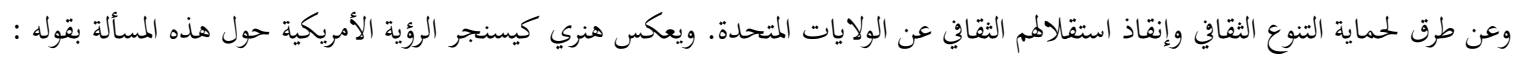

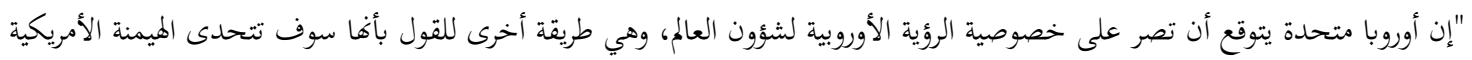

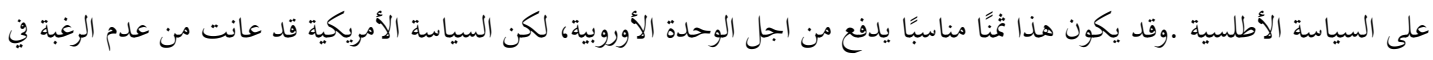

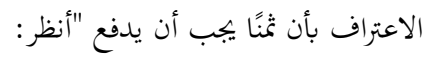

Henry Kissinger, The White house Years Boston: Little brown and Co, 1979), 82.

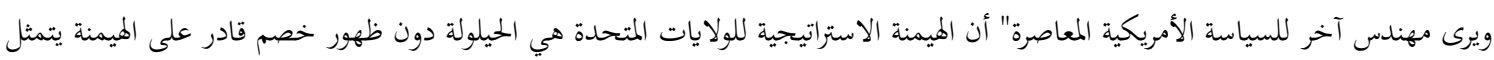

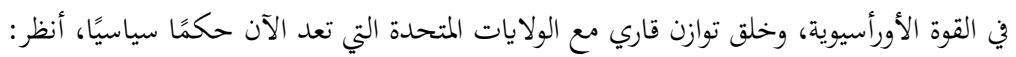


ونظرا لتداخل الغرب في العالم الإسلامي منذ أوائل القرن التاسع عشر على الأقل، فإن الغرب كظاهرة

لا يزال يحير الفكر الإسلامي الحديث. ومنذ بدء الاستعمال في أوائل القرن التاسع عشر تساءل المفكرون المسلمون "ما الغرب؟" فقد مثل الغرب تحديا أساسياً للعقل العربي الإسلامي الحديث، وهذا التحدي هو الذي أرغم الفكر الإسلامي الحديث على نقد الماضي ومحاولة انتحال روح الغرب العلمية الحديثة، مع ذلك بقي الغرب مصطلحا غير محدود في الفكر الإسلامي الحديث: هل يمثل الاستعمار؟ أم الليبرالية؟ أنام المسيحية؟ أم الرأسمالية؟ أم الاشتراكية؟ وكان الغرب باعتباره كيانا علميا وثقافيا اجتماعيا باستمرار بالنسبة للعقل المسلم هجوميا عدوانيا. لقد كان مفكرو النهضة في العالم العربي والمفكرون الليبراليون في الهند المسلمة في القرن التاسع عشر 39 تأسرهم الإمكانات المختلفة التي يقدمها "العقل الغربي" والعلم الغربي وكانوا واعين بالركود العميق لمجتمعاقم وان هذا الركود ينتهك مبدأ إسلاميا رئيسا وهو "مصلحة الأمة"، وسعوا لتحسين الأوضاع بإحياء هذا المبدأ وربطه بالحاجة إلى العلم الغربي. بعبارة أخرى، فإن العلم الغربي ومبدأ المصلحة العامة لا يلتئمان في نظر نخبة الفكر في عصر النهضة، وعلى النقيض من ذلك، يجب للوفاء بالمبدأ الإسلامي تبني منطق الحداثة (مثلا العلم)، 40 وهكذا رأى رفاعة الطهطاوي ومحمد عبده وسيد احمد خان وأقرافم أنه يمكن حل هذا التوتر (الركود مقابل العلم) فقط، إذا ربطت نخبة الفكر في وزمافم فلسفتها المذهبية بمنطق الفلسفة والعلم الغربيين؛أي تأييد التقدم، وهو المفهوم المحوري لحداثة القرن التاسع عشر الأوروبية ومع ذلك، فقد وضح أثناء محاولة حل هذا التناقض تناقض آخر: تناقض العلم والاستعمار وكلاهما من معطيات الغرب. كان مفكرو

Zbigniew Brezezinki, The grand Chessboard: American primary and it Geostratejic Imperatioes

(New York: basic Books, 1997), Especially Chapter Two.

39 A. Ahmad, Islamic Modernism in India and Pakistan (London: Oxford University Press. 1967).

$$
\text { لمناقشة مفصلة لهذا الموضوع أنظر: }
$$

Ibrahim M. Abu-Rabi, Intellectual Origins of Islamic resurgence on the Modern Arab World (Albany : State University of New York Press, 1996). 
القرن التاسع عشر المسلمون واعين بشكل جيد أن الغرب لا يعني بساطة: العلم، بل والسلطة العسكرية والسياسية أيضا.

حددت التوترات المزدوجة بين الركود الإسلامي والعلم الغربي وبين العلم الغربي والهيمنة الغربية معالم

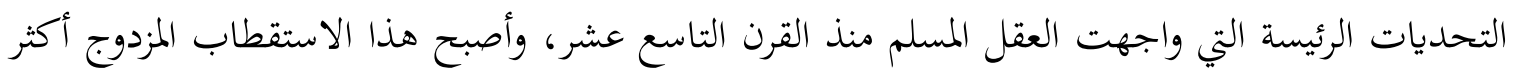
تعقيدا في منتصف الخمسينيات بعد غاية الاستعمار الرسمي في أغلب الدولة الإسلامية. ومستت الدولة

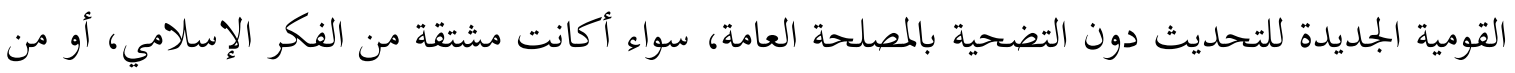
الفكر القومي، وسعت للاستقلال الاقتصادي والاجتماعي عن الغرب، الذي كانت ولا تزال تعتمد عليه،

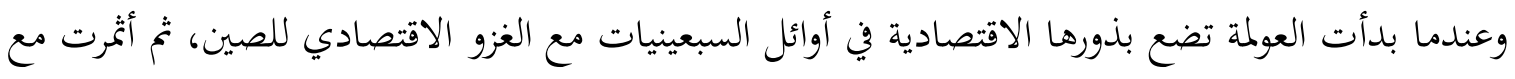

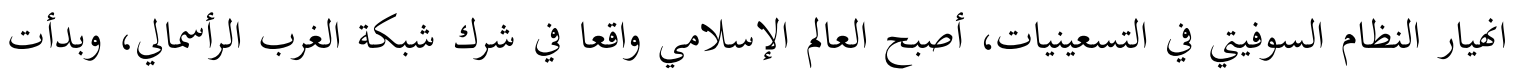
الفجوة في القوة بين العالم الإسلامي والغرب في الاتساع إلى درجة أن دولة مثل سوريا كانت تستخدم الاتحاد السوفيتي لدعم مركزها، استرضت الغرب بإرسال قواقا لماربة العراق وتققت النظم السياسية الإسلامية توا

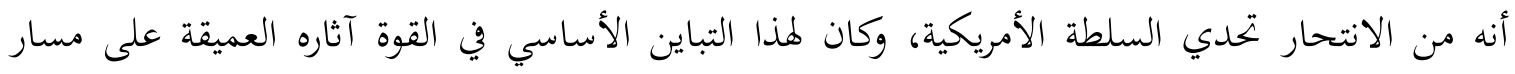

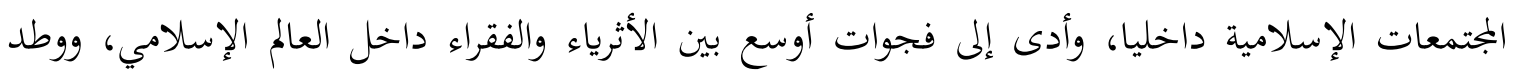
التحول الدولي في مركز النفوذ بدورة النخب السياسية والعسكرية المسلمة.

\section{الاستقلال الثقافي في ظل العولمة: - الم}

ورثت الدولة القومية الإسلامية الحديثة عالما من التناقضات، فعلى حين كانت تسعى للتحرر من

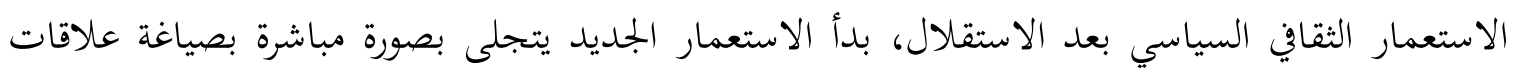

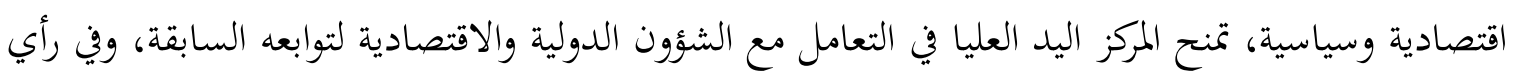

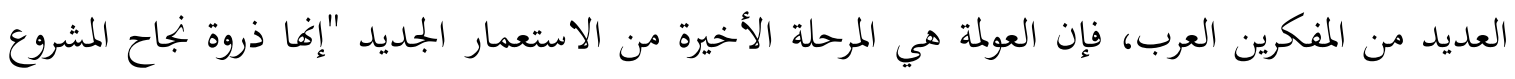


الرأسمالي العالمي النطاق". 41 فقد سمح الاستعمار الجديد للمركز بالحفاظ على أسواقه ونفوذه الثقافي وأحيانا

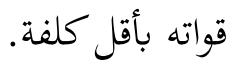

وعلى حين يمكن ممارسة القياس الكمي للعوامل الاقتصادية، يصعب قياس الثقافة بنفس الطريقة، فقد خلفت غاية الاستعمار الرسمي ركاما ثقافيا معقدا لايمكن التغلب عليه بين عشية وضحاها، ووجدت نخبة الفكر في النظام القديم، التي حاربت الاستعمار السياسي، دون الثقافتين وجدت نفسها في مراكز مسيطرة، واقعة بين الثقافة الغربية التي تبنتها وثقافة البلد الأصلية، ودعم الذين آثروا التعريب- كما في شمال أفريقياالتنوع الثقافي والاستقلال عن ثقافة الغرب المسيطرة.

إن نظام ما بعد الاستعمار - توأم الاستعمار الجديد الذي ظهر منه- لهو نتاج النظام العالمي الجديد. 42 وغالبا ما يستتبع الاستعمار كحدث تاريخي ذي أهمية قصوى احتلال قطر (أوربي) لقطر آخر عسكريا، والسيطرة المباشرة على الموارد الطبيعية، مما يكون له آثاره على المصالح الاستراتيجية للمستعمر. ولقد سعت النخبة السياسية للدول القومية الجديدة إلى تحديث بلادها من خلال التقليد العمى للغرب، واستفادت من الحماية السياسية والعسكرية التي أسبغتها الغرب عليها، وعلى سبيل المثال لم يثر اغرب قط في بعض هذه الأقطار مسألة حقوق الإنسان ولا غياب الديمقراطية؛ لأن مجمل اهتمامه كان موجها للحفاظ على مصالحه الاستراتيجية، فأساس الاستعمار الجديد شكل جديد من السيطرة الاقتصادية يسمح بظهور الأشكال المتمايزة الأخرى من الهيمنة خاصة السياسية والفكرية والثقافية. 43

$$
\text { 41 بلعبد الإله بلقزيز، العولمة والهوية الثقافية، المستقبل العربي (مارس 1998)، ص29 } 29 .
$$

42 "le post-colonialisme est un phenomene tres recent qui date debut des annees 1990, comme suite a la chute des regimes communists, la Guerre du Golfe etl' effritement du peu d'unite que le Tiers Monde etait parvenu a construire..Le post-colonialisme est, avant tout , le preduit dui nouvel ordre mondial," Mahdi Elmandjra, La decolonization culturelle: Defi majeur du 21 leme siecle (Marrakech [Morocco]: Editions Walili, 1996, and Paris: Futuribles, 1996), 208.

$$
\text { انظر مث مُ لا عمد عابد الجابري المشروع النهضوي العري) بيروت :مركز دراسات الوحدة العربية، 1996وكتابه بالفرنسية: }
$$

Introduction a la critique de la raison arabe (Casablanca: Editions le Fennes, 1995). 
لقد شعرت أقطار العالم الثالث، إلى حد ما، بالتخفف خلال الحرب الباردة من ضغط الغرب السياسي والثقافي؛ لأن توازن القوى العالمي سمح لها بالعمل بحرية، ومع ذلك تغير الوضع القائم باهيار النظام السوفيتي الهزيمة العسكرية للعراق، وترك هذان الحدثان العالم العربي والثقافة العببية بلا دفاعات في وجه الهيمنة الأمريكية وبظهور الولايات المتحدة كقوة عظمى وحيدة اقم المركز -ولا يزال- عددا من الدول مثل كوبا

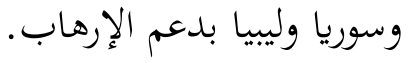

ولم يزل الهامش يعتمد على المركز منذ عصر الاستعمار، كما أسس انتشار الأفكار عبر الأقمار الصناعية علاقة جديدة بين العالم الإسلامي والغرب، ويمارس. المركز ما يمكن تسميته بـ "ثقافة امبريالية" مهيمنة. ويلفت معظم المفكرين المسلمين الذين شبوا في ظل الاستعمار أنظارنا إلى الاستنتاجات الرئيسية للمفكرين الأوروبيين، وحتى بعض الأمريكيين، حول صعود الولايات المتحدة في الأعوام الأخيرة، و تأثيره الثقافي العالمي، حتى داخل اوربا، ويقولون إن "الإمبراطورية الأمريكية هي الوحيدة في العالم وهي المهيمنة بشكل مطلق، ولأول مرة في التاريخ الإنساني تتبدّى هذه الظاهرة الملفتة للنظر وتستمر ". 44

إن الولايات المتحدة إمبراطورية فريدة، فهي المنتج الرئيس لكل أنواع السلع وهي أيضا مستهلك شره، وتاريخها ميز من بدايته الأولى بميل متطرف إلى التوسع:

"إن التاريخ الأمريكي بكامله موسوم بابحاه دائم نحو التوسع والظمأ للأراضي والظمأ للقوة، والظمأ. للجدة ولأبجاد وإلى العديد من الحاجات التي يتوجب إشباعها". 45

ووفقا لرأي الناقد الأدبي الأمريكي الذائع الصيت "إدموند ويلسون" لم يكن التوسع الأمريكي في ماوراء البحار في أعقاب هزيمة ألمانيا النازية محض مصادفة:

44 Paul-Marie de la Gorce, le Dernier Empire (Paris: Grasset, 1996), 16.

Claude Kulien, L'empire americaine (paris: Grasset, 1986), 25. The same ideas are presented by jean Jacqus Servan-Shreiber la Defi American (Pris: Deneol, 1967). 
"كما نعتقد أننا نحرر أوربا ونناضل استعمار اليابان الإقطاعية، ولكننا ظهرنا فجأة بعد الحرب (العالمية الثانية) محتلين أو مسيطرين على الأقطار الأجنبية في كل من أمريكا وأوربا وآسيا والشرق الأوسط دون ترحيب أحيانا، كما كان الفرنسيون في الجزائر أو البريطانيون في قبرص أو الروس في أوربا الوسطى، وبعد أن صدمنا استعمار الآخرين لسنوات طويلة طورنا نوعا جديدا خاصا بنا، ووجدنا انسنا نعبس في وجه الاتحاد السوفيتي، وننفق المليارات على أسلحة موجهة ضده وحتى على أسلحة كان مجرد بحريبها خطرا على سكان بلدنا ودون أية استفزازات حقيقية لنا، ولكن لسبب محدود المعقولية وهو أن نتحدى الروس السوفيت للسيطرة على أجزاء واسعة من العالم". 46

إن التجلي الحديث للعولمة في رأي العديد من مفكري العالم الثالث هو أيضا التجلي الحديث للأمركة التي تدعم نوعا جديدا من النماذج الاقتصادية والثقافية "فالعولمة إلى جانب كوغا نسقا اقتصاديا فإنها فكرانية (أيديولوجية) تخدم هذا النسق فالأمركة والعولمة متضافران بشكل بالغ". 47

يحتاج العالم الإسلامي في سعيه للتنمية إلى العلم والتكنولوجيا الغربيين، ومع ذك لا يككن استيراد العلم الغربي دون القيم الأخلاقية التي أنتجته، وقد استخدم الغرب في طوره الاستعماري الثقافة والأفكار لاستعمار العالم الثالث، ولذذا ازدهر الاستشراق وبعثات التبشير والأنشطة المشاهة، وجرى استبقاء الاستعمار التقليدي بالحضور المادي لقوات البلد الأم في ما وراء البحار، فقد تعاون الغزو المادي والفكري وأخذ كل منهما بيد الآخر إلا أن الموقف مختلف إلى حد ما في عصر الاستعمار الجديد بتقدمه السريع في التكنولوجيا، حيث تتعرض الهوية الفكرية والثقافية للأمم الصغيرة للخطر. وقد كان غرض الاستعمار إنشاء نخبة ثقافية محلية بقيم غربية، وغرس أنساق التعليم والفكر الغربية في العالم الثالث، وكانت ثقافة الاستعمار أيديولوجية بطبيعتها، واليوم أدى الغزو الثقافي من خلال التقنية المتقدمة إلى النتيجة التالية: سعت الثقافة الغربية -إضافة إلى كوغا

\section{: $\quad 46$}

Edmund Wilson, The American Earthquake: A Chronicle of the Roaring Twenties the Great Depression and the Dawn of the New Deal (New York: Da Capo Press, 1996), 569.

$$
\begin{aligned}
& 47
\end{aligned}
$$

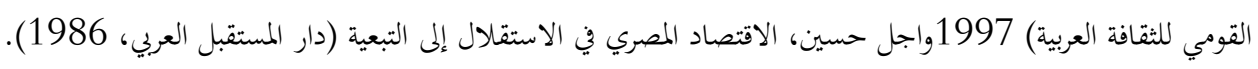


أيديولوجية بطبيعتها- إلى قهر وسائل النقد والعقلانية في العالم الإسلامي وفي حالتنا استهدفت العقل العربي والإسلامي محاولة جعله ينسى ماضيه المتفرد والمجيد.

إن أحد حقائق عالم اليوم التي يؤسف لها أنه ليس ثمة من يمكنه منافسة الهيمنة الاقتصادية والفكرية الأمريكية، فهذا البلد يمتلك موارد اقتصادية ضخمة وتقنية متقدمة ومهارة عسكرية كما يملك الإرادة لغزو العالم بكامله فكريا، 48 ولذا نعيد صياغة السؤال الذي سبق طرحه على الوجه الآتي: ماذا يجب عمله لتحقيق هضة ثقافية وسياسية واجتماعية عقلانية في العالم الإسلامي المعاصر؟ إن الفكر الإسلامي لا يمكنه بتحنب الانعكاسات الكاملة لتحدي الغرب الثقافي المعاصر وبالأخص تحدي الولايات المتحدة في سياق النظام

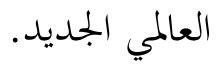

فلقد أصبح النظام العالمي الجديد الذي تدشن في أعقاب المزيمة العسكرية للعراق ظاهرة سياسية عالمية النطاق، وعلى سبيل المثال فقد اتخذ رد فعل القوميين في العالم العربي على الغرب شكل تكيف أساسي مع الأوضاع بعد غاية الحرب الباردة، وفقد معظم الدول الاهتمام بالوحدة العربية، ويرتبط بهذا السياق ما قاله محمد عابد الجابري أحد المفكرين المغاربة من ذوي التوجه القومي العربي:

إن الوجود العربي حي، وقد أظهرت الحرب القريبة التي شنها الحلفاء على العراق، بجلاء، حيوية العروبة، وحضورها الكامن في روح الجماهير العربية التي احتشدت لتأييد العراق وغالبا ضد رغبات حكوماقا. ومما له دلالته أيضا في هذا الصدد الموقف العربي القومي الذي اتخذه مفكرو الفرانكفون المغاربة الذين حرم العديد منهم من تعلم العربية في طفولتهم. فبالإضافة إلى تعبئة تأييدهم وراء العرب فقد عبروا عن فزعم وإحباطهم من الموقف الأوروبي من العراق. 49

وفي عصر الإمبريالية كان الغرض الأساسي للمشروع القومي العربي هو تحقيق استقلال العالم العرب، وساعد استقلال الجزائر على تحقيق هذا الهدف ولكن حرب عام 1967 كانت نكسة رئيسة بمنعها الوحدة والتضامن العربين من أن يمدا جذورهما الفكرية والتنظيمية في المجتمع. 


$$
\begin{aligned}
& \text { إن ظهور الدولة الإقليمية في العالم العربي كحقيقة سياسية دولية وكوضع نفسي واجتماعي /اقتصادي } \\
& \text { لا يمكن تحاوزه، وضع مزيدا من العقبات في طريق المشروع القومي العربي، فالعالم العربي منقسم الآن على } \\
& \text { نفسه بشكل يفوق أي وقت مضى، وعلى العالم العربي أن يأخذ في الاعتبار - إضافة إلى التحدي الثقافي } \\
& \text { والانقسام السياسي- المشروع الصهيوني والتغيرات الحاد التي طرأت على طبيعته في سياق النظام العالمي }
\end{aligned}
$$

$$
\begin{aligned}
& \text { ويمكن القول تأسيسا على الحجج السالفة، إن المهمة العاجلة للعالم الثالث هي السعي للتحرر } \\
& \text { الاقتصادي والسياسي من هذه الميمنة الجديدة، وأحد العوامل الحاسمة في ذلك هو التخلص من الاستعمار } \\
& \text { الثقافي لأن الهدف رالأساسي لما بعد الاستعمار هو الهيمنة الثقافية وبث القيم الغربية في العالم الثالث، } 50 \\
& \text { ويرفض الشمال إجراء حوار شامل حول القيم الثقافية مع الجنوب، مفترضا أن قيمه الخاصة هي المعيار؛ أي } \\
& \text { أها قيم عالمية وان تبنيها سيحل مشكلات العالم الثالث الاقتصادية والاجتماعية، } 51 \text { وبغض النظر عن قدرات } \\
& \text { الغرب العسكرية والنووية الفائقة، فإن مابعد الاستعمار سلاح يهدف إلى تدمير التنوع الثقافي } 52 \text { في عالم } \\
& \text { اليوم وخلق ثقافة عالمية متجانسة واحدة هي "الثقافة المغربة". } 53
\end{aligned}
$$

وليس مصادفة أن معزظم المناطق الدولية الساخنة تقع في العالم الإسلامي، والسبب الرئيس في ذلك

رفض الغرب التوصل إل تفاهم مع أي نسق للقيم سوى نسقه، ومع ذلك فإن العوامل الخارجية ليست

$$
\text { وحدها سبب المشكلة، فاعالم الإسلامي - شأنه شان أغلب العالم الثالث- يعاني من غياب الديمقراطية، }
$$

50 "Mais la liberation du Sud passe d'abord par une decolonization culturelle carun des principaus objectifs du post-colonialisme est l'hegemonie culturelle et la propagation des valeurs occidentntales, les contlits a venire seront des confits valeurs et il y a une tres frande urgence a developer une communication culturelle entre le nord le Sud" M.Elmandjra, La Decolonisation culturelle, 214.

51 "Le Nord a deploye jusqu'a present tres peu diefforts por compredre et encore moins pour parler le langage du Sud. II faut accorder une priorite aux systems de valeurs pour se render compte que la crise actuelle entre le Nord et le Sud est une crise du systeme total," mahdi Elmanjra, Retropecive des futures (Casablanca: Ouyoum, 1992), 164.

52 Emandjra, La Decolonisation cuklturelle, 215.

$$
\text { المهدي المنجرة، الحرب الحضارية الأولى (الدار البيضاء: عيون،1994)، ص21 -22. }
$$


وأزمة في حقوق الإنسان، ونقص القنوات الديمقراطية التي يمكن للناس أن يعبروا عن آرائهم خلالها بحرية. ولم ينتج عن هذا الوضع أي احتجاج من الغرب، فإنتاج السلاح في ازدياد في ظل العولمة، ومستهلكوه الأساسيون في الجنوب، في إفريقيا والشرق الوسط وأفغانستان والحروب الأهلية في ازدياد، بصفة أساسية، في الجنوب، فقد تعلم درسا هاما بعد الحرب العالمية الثانية وهو ألا تنشب أية حروب في أوربا- بما فيه الاتحاد السوفيتي المنحل والشمال عامة.

ومن المعضلات الرئيسة التي تواجه العالم الإسلامي المعاصر حماية التنوع الثقافي العالمي والتعددية في وجه لاالهيمنة الأمريكية المتصاعدة ويكمن حلها في تققيق تغير رئيسي في تفكير كل من الشمال والجنوب، فعلى الشمال أن يعترف بالتنوع الثقافي، وعلى الجنوب تأكيد استقلاله الثقافي، ووفق رأي أحد إسلاميي شمال إفريقيا فإن "التحرر من الاستعمار الثقافي مهمة أجيال عديدة". 54

لقد بدأت الولايات المتحدة في تصعيد الحرب ضد أعدائها العالميين الجدد في أعقاب اهيار الاتحاد السوفيتي، وجرى التعبير عن هذا بوضوح في أطروحة "صمويل هنتجتون"55 وأصبح الغرب مشغولا بخطر الإسلام أو الإرهاب الإسلامي أو الأصولية، وجرى تحجيم حركة العالم الإسلامي وتفتيته بكل تركيبه الثقافي والعرقي إلى ذرات، وجرى بحاهل حقيقة أن أغلب المسلمين يعيشون في أقطار تحكمها نظم تسلطية يدعمها الغرب وديمقراطياته. 56 إن التخلص من الاستعمار تبدأ النخب الفكرية في العالم الثالث وفي الغرب جديا النظر في أن التحديث يجب أن لا يعني التغريب وان هناك طرقا غير غريبة للتحديث.

إن أحد الظواهر الأخاذة التي تسمم الحضارة الحديثة هي التحول العميق الذي حدث لدى تحول الحضارة من ثقافة الإنتاج إلى ثقافة المعلومات والمعرفة العلمية، وقد أمطكن ذلك بسبب الطفرات الجذرية في

54 Elmandjra, La Decolonisation Culturelle, 15.

Samuel Huntington, The Clash of Civilization.

56 Brian Coodwin, How the leopard Cahnged its Spots: The Evolution of Complexity (New York: Charles Scinbner's Sons, 1994); Mitchell M. Waldrop, Complexity: The Emerging Science at the Edge of Order and Chaos (New York: Simon and Schuster, 1992). 
العلم والتقنية. ويسبب تفوق الغرب العلمي أصبحت فجوة المعلومات بين الشمال والجنوب لايمكن تخطيها. بل واستمرت في الاتساع يوما بعد يوم، وعلى سبيل المثال كانت الولايات المتحدة تملك 56\% من إجمالي بنوك المعلومات في العالم وخصوصا بنوك البحث والعلم، بينما تمتلك الجماعة الأوربية 28\% واليابان 12\% والعالم الثالث 1\%

واستفحلت الفجوة المعلوماتية بين الشمال والجنوب في عصر العولمة بفعل هذه "القوة الرخوة" التي تعرف بأها قوة التحكم والاتصال، وإدارة الحرب التكنولوجية عن بعد. ووفقا لما يقوله "رتشارد فولك" من جامعة يرنستون فإن تفكير النخبة الأمريكية والغربية الحالي يتجه إلى أن إمكانات علم التحكم والاتصال غير محدودة.

ويحلم المشتغلون بالتخطيط الاستراتيجي في الولايات المتحدة بالسيطرة على شبكات التحكم والاتصال والثروة الأسطورية الناتجة عن الصناعات الفكرية للتعلم والمعرفة، ويحلمون ببناء إمبراطورية العصر الإلكتروني الجديد، التي ستمد السوق العالمي وهو في مركزها بجهازه العصبي بفضل تقنيات المستقبل.57 إن بلدا بقوة شباب الولايات المتحدة ما يزال مفتونا بغزو المهاجرين الأوائل لقلب المروج الأمريكية. في البداية بدت مساحات الأراضي التي يجري غزوها غبر محدودة، ولكن مع فاية القرن التاسع عشر توقف هذا التوسع الإقليمي، وكان على العقل الأمريكي أن يحاول فتح آفاق جديدة.: كانت الآفاق الجديدة للنظام العالمي الجديد هي غزو الفضاء، الذي يعني النفاذ الكامل إلى موارد السيبرنت، أي كل ما يتعلق بتقنيات التحكم والاتصال، ومع استمرار نزيف العقول من الدول الفقيرة الصغيرة وهجرة العديد من خبراء التقنية العالية من الاتحاد السوفيتي، امتلكت الولايات المتحدة موارد تكنولوجية هائلة، وبدت العولمة الأمريكية بشكل أكثر جدية58 مع ملاحظة أن المركز المتقدم شجع هجرة الفنيين المهرة من العالم الثالث، وبدأ سن القوانين التي بتعل الهجرة أصعب كثيرا للمهاجرين من غير المهنيين. 59

57 Richard falk ": Vers une domination mondiale de nouveau type" le Monde Diplomatique (May 1996), 16. 


$$
\begin{aligned}
& \text { لقد أدى هذا إلى تفوق الغرب السياسي والاقتصادي، وانتشار الأفكار الغربية من خلال اكتساب } \\
& \text { التقنيات الغربية، ونزيف العقول من العالم الثالث إلى المركز المتقدم تكنولوجيا، وتسبب هذا كله في غياب } \\
& \text { حوار ثقافي جاد بين الشمال والجنوب، "ومن ناحية أخرى أسهم غياب الاتصال والتسامح الثقافي في } \\
& \text { تعريض السلام للخطر لأعوام قادمة". } 60 \\
& \text { وعلى الرغم من التفوق المعلوماتي الذي ظهر به الغرب فما تزال شعوبه لا تعلم إلا أقل القليل عن } \\
& \text { شعوب العالم الثالث والتحديات التي تواجهها، فالإعلام الجماهيري الحالي في المركز المنتصر لا يفضل نظيره } \\
& \text { منذ عشرين سنة؛ إذ ما يزال يشن حربا من التجاهل للمشاكل الحقيقية المؤثرة في الجنوب، ويصبح هذا الوجه } \\
& \text { المزدوج للعولمة (معلومات كثيرة عن العالم الثالث ومعرفة قليلة جدا عن مشاكله) أكثر خطورة عندما تتغلغل } \\
& \text { القوى الرأسمالية الجديدة في كل ركن م العالمهة وتصوغه وفقا لمتطلبات الاقتصاد العالي الجديد61 ويستخدم } \\
& \text { الغرب تقنياته البحثية المتفوقة لجني أرباح أكثر على حساب العالم الثالث وبالأخص مع استمرار الظروف غير } \\
& \text { المتكافئة بين الشمال والجنوب. }
\end{aligned}
$$

Joseph Nye et William Owens, "American's Information Edge" foreign Affairs (MarchApril 1996).

59

S. Sassen, Losing Control? Sovereignly in an Age of Globalization (New York: Comumbia University Press, 1996).

$$
60
$$

J Nye and W. Owens "Ameriva's Information Edge" 29-30.

$$
61
$$

William Geider, One World, Ready of Not: The Manic Logic of Global Capitalism (New York: simon and Schuster, 1997); A. Gresh, "Les Aleas de K'internatioalisme" Le Monde Diplomatique (may 1998), 12.

انظر

Richard Banet and John Gavanagh, Global Dreams: Imperial Corporations and the New York Order (New York: Somon and Dchuster, 1994): M.K. Bouguerra, Le Recherche contre kleTiers-Monde (Paris: Puf, 1993). 
عجلت العولمة أيضا نزيف العقول من لعالم الثالث إلى المركز المتقدم ويفضل العديد من مهنيي ومفكري العالم الثالث الإقامة في الولايات المتحدة، وأطلق على هذه العملية بحث، "النزيف الفكري" لأهما تستنفذ الخبراء من الأقطار الفقيرة التي تحتاجهم أشد الاحتياج في كل الحقول العلمية.

ولا يسعى العديد من المهاجرين لمستويات اقتصادية واجتماعية أفضل فقط، بل يهاجرون أيضا لأن العملية الإنمائية في أقطارهم الأصلية ينقصها الرؤية السليمة لإدماجهم بشكل خلاق، ويكمل ذلك غالبا التقليد الأعمى للشمال الحديث، بما يؤدي لنقل التكنولوجيا، دون أي إسهام خلاق من الجنوب، بعبارة أخرى يمكن للجنوب أن يشتري التكنولوجيا، ولكن يجب عليه أن يبتدع أشكاله الخاصة من الحداثة والتحديث. ولايمكن إبداع هذه الأشكال إذا ما استمر رحيل المهنيين المهرة، وكان من تداعيات هذا الوضع البائس معاناة العالم الإسلامي، وبقية العالم من المشاكل المتداخلة من الأمية وغياب البحث العلمي الصارم ونقص القيم الديمقراطية.

إن نزيف الموارد الفكرية ظاهرة تنذر بالخطر، إلا أها أدت إلى نتائج إيجابية، وبالأخص، في المركز المتقدم حيث يدور الحوار حول القيم الدينية والثقافية المتنافسة بين أناس من جماعات دينية وثقافية مختلفة، 63 وإذافة إلى ذلكك يحتاج الشمال إلى عنمال العالمؤ لاثالث، فتستورد أوربا العمالة الماهرة وغير الماهرة من العديد من أقطار العالم الثالث وأبرزها المغرب والجزائر وتونس وتركيا وباكستان. وتكشف هذه الهجرة الجماعية المستمرة عن التخلف المستمر لهذه الأقطار، فالتخلف هو عدم القدرة على إبداع واستخدام الموارد الإنسانية الماهرة ببراعة وعقلانية، 64 ولا يرحل المهاجرون المهرة عن أقطارهم للأسباب المالية وحدها، بل لأن البلد الذي يتبناهم يقدم فرصة أفضل للحصول على تعليم أعلى في مهنتهم وفرصا أكبر للتقدم.

إن المعرفة العلمية معلم رئيسي على التنمية والتقدم في المجتمع المعاصر. ونظرا للتقدم السريع في العلم والتكنولوجيا فإن عالم المعرفة سيضاعف معلوماته العلمية في العقد القادم أو قريبا من ذلك، وهذا قدر هائل

Claude liuzu, Race et civilization: L'Autre dans la culture occidentale (Paris: Syros, 1992)

Elmandjra. La Decolonisation culturelle, 175. 
بالنظر إلى حقيقة أننا نتعلم في عقد أكثر مما كان أسلافنا يتعلمونه في ألف عام، إلا أن الغرب واليابان يتحكمان في أغلب هذه المعرفة بينما تستمر أفضل عقول العالم الثالث في الهجرة إلى المركز، ويتسبب هذا في فجوات معرفية أساسية بين الشمال والجنوب تجعل -بتزاوجها مع إحساس الشمال بسموه الثقافي- من الصعب تأسيس اتصال ثقافي بين الشمال والجنوب. "فمسألة التعصب للعرق هي التي بتعل من الصعب تأسيس اتصال ثقافي حقيقي"65 عن الغرب الذي يشكل الآن 22\% من سكان العالم، وربما 16\% فقط بعد ثلاثين عاما يستهلك 70\% من إجمالي موارد العالم وتسيطر عليه ثلاثة هواجس: الاتجاهات السكانية للعالم الثالث والأخلاق المسيحية اليهودية واليابان. 66

يواجه العالم تحديث التعددية لاثقافية ويحمل المستقبل مشهدين ممكنين: إما إعادة تعزيز نسق دولي أحادي، كما يبدو اليم في النظام العالمي الجلديد المتميز بالتفوق الأمريكي اةو الحفاظ على التنوع الثقافي، وهي القيمة التي يجب على العالم الدفاع عنها، وبدون هذا الدفاع لن يحقق العالم الثالث تحرره الثقافي الكامل وستقلاله. عن الغرب يعيد خلق التاريخ المنصرم في شزكل جديد من "إعادة الاستعمار" وهو ما يسمى "مابعد الاستعمار"، وتنشى العولمة نظاما عالميا تأخذ مجراها بالفعل : فهناك اهيار الاشتراكية، وانتشار الخصخصة في أقطار مثل الصين والهند ومصر، وصعود القوى الإقليمية كالجماعة الأوربية على أثر التفوق الأمريكي، واتساع الفجوات الاقتصادية والاجتماعية بين الفقراء والأغنياء داخل الأقطار وفيما بينها، وعولمة الاستغلال متداع طبيعي للخصخصة، والاستثمار متعدد الجنسية، وصعود القومية المغالية، والتطهير العرقي، ومشكلات اللاجئين الجديدة، وإضفاء الطابع الدولي على الجريمة، وخاصة ذات الصلة بالمافيا، وزعزعة الدولة القومية، وخلق أعداء دوليين جدد. 67

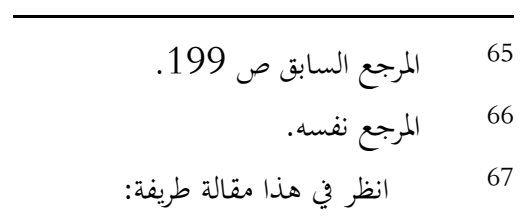

Le Sous-Commandant Marcos, "La 4e guerre mondiale a commence," Le Monde Dimplomatique (August 1997). 


\section{الصهيونية والعولمة:}

اضطلع المشروع الصهيوني في مابعد الاستعمار بدور جديد تدعمه الولايات المتحدة ماليا ومعنويا، وساعدت الدول الاستعمارية السابقة في عبور مستعمراها من إلى عهد مابعد الاستعمار وأقرته الأمم المتحدة، وسعى المشروع الصهيوني -إزاء هذا السياق - لاعتراف الدول العربية الرئيسية به.

أعاد المشروع الصهيوني، الذي بدأ في الوقت مع المشروع القومي العربي في القرن التاسع عشر أعاد ابتكار نفسه في ظل حكومة نفس الليكود، بالتشبث العنيد بثنائيات التحضر ضد الهمجية، والرحمة مقابل الإرهاب، والديمقراطية مقابل الشمولية، وإسرائيل الديمقراطية مقابل العرب والمسلمين المتخلفين.

إن ما سيجري للمشروع الصهيوني في المستقبل مسألة هامة، فعلى سبيل المثال، يستحيل تقريبا توقع ما ستكون عليه إسرائيل بعد خمسين عاما من الآن بدقة تامة، ولكن مع بدء العولمة في مد جذورها في الاقتصاد والثقافة العالميين، سيصبح الشرق الوسط عموما وإسرائيل خصوصا أقل أهمية مما هما عليه الآن بالنسبة لاستراتيجيات المركز الرأسمالي العسكرية والسياسية، فالبترول (العربي) أو الصهيونية، أو أي شكر م أشكال القومية في الشرق الوسط، سيتوجب عليه تغيير سماتما بشكل حاد في مناخ التغيرات السياسية والاقتصادية والثقافية العميقة الناجمة عن العولمة، ويعني هذا انه يستحيل رؤية مصير إسرائيل بعد خمسين عاما دون الأخذ في الحسبان أهمية الشرق الأوسط ككل في الفترة نفسها.

ولقد شهد عام 1948 ذروة انتصار المشروع الصهيوني بإنشاء دولة إسرائيلية قوية هدفها- كما هو

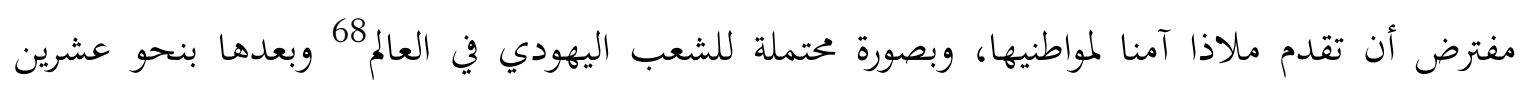
عاما، ظهرت إسرائيل كأقوى دولة عسكرية في الشرق الوسط بفضل المزيمة الكاملة للناصرية عام 1967، وتدمير قدرات العراق العسكرية وفي حرب الخنليج الثانية وأما أعقبها. وعلى الرغم من اجتذاب إسرائيل سكانا مختلفين من أركان العالم (على سبيل المثال العالم العربي وأوروبا الشرقية والاتحاد السوفيتي السابق وإثيوبيا)، فإن أغلبية يهود المركز في غرب أوربا وأمريكا الشمالية ولم يبدوا حتى الآن أي اهتمام حقيقي

68 See Zeev Stemhell, the Founding Mytrhs of Israel: Nationalism, Socialism and the Making of the Jewish State (Princeton: Princeton University Press, 1998). 
بالهجرة إلى إسرائيل أو العيش فيها، وبمعنى آخر فإن المشروع الصهيوني لم يتحقق، وبقي مبتورا، على الرغم من التضحيات المالية الضخمة التي تكبدها يهود المركز لصالح إسرائيل، وبمعنى آخر يكره يهود المركز الذين استمروا في طليعة العولمة - بطبيعتهم الجوهر القومي والمحدود للصهيونية، وبرغم أفم أكبر مؤيديها في هذه المرحلة من التاريخ فقد يعادوها في المستقبل. وقد منع العديد من العوامل الهجرة المكثفة ليهود المركز إلى إسرائيل منها: طبيعتها العسكرية، فقد دخلت إسرائيل عدة خروب رئيسية منذ عام 1948، وطبيعة المشكلة الفلسطينية التي ظهر في أعقاب تأسيس إسرائيل، يقل التأكد شيئا فشيئا في العالم الغربي حول المعتقدات المحورية للصهيونية وبالأخص في ظل حكم الليكود.

وفي تقديري ستكون الخمسين سنة القادمة في عمر إسرائيل مختلف نوعا عن الخمسين الماضية، فثمة استياء بين العديد من الإسرائيليين ويهود ما وراء البحر من الطبيعة العسكرية للدولة، وتقدير أكبر متزايد لمعاناة الفلسطينيين، وتفهم عميق، حتى من أكثر اليهود صهيونية لعدم قدرة الاقتصاد الإسرائيلي البقاء بشكل دائم على المساعدات الأجنبية، سواء كانت أمريكية أو أوربية أو حتى يهودية.

إن مصير الفلسطينيين والإسرائيليين سيصبح أكثر تداخلا نظرا لعوامل ثقافية وسكانية: فعلى الجانب السكاني، يشير معدل مواليد السكان اليهود والفلسطينين الحالي إلى أن الفلسطينيين، الذين يحملون المواطنة الإسرائيلية في إسرائيل، والذين يقدر عدهم إلى ثمانمائة وخمسين ألفا سيصلون إلى حوالي مليونين ونصف المليون بعد خمسين عاما مع استبعاد أولثك الموجودين في الضفة الغربية وقطاع غزة الذين يقدر عددهم بعد خمسين عاما بحوالي من 5-6 مليونا، ومن المشكوك فيه أن يزيد عدد السكان اليهود في إسرائيل بعد خمسين عاما عن تسعة ملايين.

وعلى المستوى الثقافي سيكون لليهود المهاجرين من العالم العربي كلمة أكبر في الشؤون السياسية والثقافية في المجتمع الإسرائيلي، وسيؤيدون -بغض النظر عن ميولم السياسية المحافظة- تقاربا ثقافيا عربيا وإسرائيليا، وهذا أمر عادي بالنظر إلى أن العديد منهم يحاولون إعادة اكتشاف هويتهم الثقافية والدينية التي تم بناؤها خارج نطاق الحدود الإسرائيلية/الفلسطينية القائمة. 
وسيساعد كل ما سبق على إضعاف الطبيعة العسكرية القوية لإسرائيل، وسيخلق مجتمعا إسرائيليا أكثر إنسانية وابتحاها نحو الشرق الوسط، ججتمعا يرى استمرار بقائه في التوافق مع بيئه الشرق أوسطية

والثقافية العربي الإسلامية عامة.

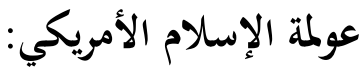

أود أن ألمس نقطة رئيسية أخيرة تعلق بحياة المجتمع المسلم في الولايات المتحدة، فكثير من المسلمين الأمريكيين يعتقد أن هذا البلد وحده هو الذي تتاح فيه للمسلمين فرصة حقيقة لتشكيل هوية إسلامية أصيلة ووعي ورؤية إسلامية للعالم، وهذا الوضع مبني على مايلي:

1- يتسامح الحيز الثقافي والديني الأمريكي، بعد أن تطور عبر الزمن، مع تكوين هويات دينية وثقافية

جديدة.

2- وما كان التكوين الثقافي للمجتمع الأمريكي المسلم في غاية التنوع، فإن هذا البلد أحد المناطق القليلة في العالم التي تمكن للمسلمين القادمين من خلفيات ثقافية وعرقية متنوعة من الاختلاط والتمازج مع بعضهم بعضا. مما يمهد الطريق لتكوين ثقافة إسلامية عالمية ذات سمات أمريكية فريدة وفي حدود العلمانية. وبعبارة أخرى يتعلق البعض بالرؤية القائلة بأن المسلمين في قلب الغرب المتعولم يملكون فرصة أفضل لبناء مجتمع إسلامي عالمي حقا أكثر مما لو كانوا في أي قطر آخر، وهذه أطروحة جذابة تتطلب تفكيرنا العميق. إن الأحداث الداخلية في حياة مجتمع المسلمين الأمريكيين (المحليين منهم والمهاجرين)، والتغيرات الأخيرة في النظام العالمي، وبالأخص اهيار الاتحاد السوفيتي والتورط الأمريكي في شئون العالم الإسلامي السياسية والاقتصادية حول المجتمع المسلم من مجموعة غريبة وأحيانا مروعة من الناس إلى ججتمع متميز آخذي في الاتساع، فلا يستطيع، المسلون والحال كذلك البقاء خارج الحياة الدينية الأمريكية، بعيدين عن التحديات الحقيقة التي تفرضها العلمانية على العقل المتدين. 
لايزال المجتمع الأمريكي المسلم يضع أسسه الفكرية (أي الدينية) والمؤسسية باللجوء إلى التراث الإسلامي الموري، الذي تطور في القلب الإسلامي في الشرق الوسط عبر قرون عديدة، وبالتفاوض-وإن كان بشكل واهنة وبرؤية غير واقعية أحيانا- مع الحداثة الأمريكية من أجل مكان للإسلام في هذه الثقافة، ويعلم المسلمون انه لابد للبقاء في هذا البلد، وللحفاظ على هويتهم من ظهور بجموعة من نخبة الفكر، تعيد تفسير التراث الإسلامي الضخم في ظروف جديدة، في الوقت الذي تقوم فيه بتحليل تعايش المجتمعات الدينية الأخرى مع النظام السياسي والاجتماعية الراهن.

وعلى المسلمين ان يدركوا أغم يعيشون في مجتمع الثقافات يقوم على التعددية والتنوع الديني، وأن بعض الصياغات القانونية التقليدية "كدار الحرب" و"دار السلام" لا تناسب وضعهم في المجتمع الأمريكي، وأخيرا على المسلمين بمرور الزمن صياغة مفاهيم قانونية ودينية جديدة للتعبير عن الطبيعة الفريدة لمجتمعهم، وتفاعله مع الجُتمعات الدينية الأخرى، وتسامحه مع العديد من الرؤى والفلسفات المتنوعة. بعبارة أخرى يجب على المسلمين الرد على النقد التالي الذي يشترك فيه بعض مراقبي الإسلام في الساحة الأمريكية.

"إن المسلمين الذين يأخذون دينهم بجدية لا يمكنهم الإيمان بالفصل بي الدين والدولة، والتعددية الدينية مفهوم غريب على المجتمع الإسلامي المثالي، فيجب إذن السماح للمسلمين في الولايات المتحدة بممارسة دينهم، ولكن ليس إلى الحد الذي يهدد أو يمنع حرية الآخرين الدينية، وتأكيدا ليس إلى درجة مارسة الجهاد (الحرب المقدسة) ضد غير المسلمين". 69

تأتي هذه الصياغة من مراقب معاد، ولكن الأسئلة الأهم هي كيف يمكن للمسلمين تفسير قديد العولمة الأمريكية الجديدة لكل من العالم الإسلامي والجماهير الفقيرة في الولايات المتحدة.؟ وهل استخدم المسلمون الأمريكيون كأدوات في أيدي العولمة لإفقار العالم الثالث؟ إن أحد طرق تشكيل إجماع إسلامي حول هذه الأسئلة هو تحقيق درجة من التفاهم الحقيقي بين المجتمعين الكبيرين اللذين يشكلان الإسلام

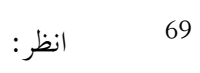

Dinesh D'Souza "Solving America's Multi Cultural Dilemma, "The World and (January 1996), 35. 
الأمريكي: المهاجرين وأبناء البلد الأصليين. وتواجه كل من المجموعتين تحديا مشتركا وهو كيفية إيجاد وعي إسلامي يمكنه إدراك المعتقدات المحورية لكل من الحداثة والعولمة، ويعي آثارها على العالم الإسلامي المعاصر. ويمكن علاوة على ذلك إثارة السؤال عن كيفية تشكيل طبقة مفكرة أمريكية مسلمة تتصف بالأصالة في انتمائها لتراثها الإسلامي الأوسع، ومهتمة بشكل قوي بالأسئلة والقضايا التي تواجه قطاعات المجتمع المسلم المتنوعة، هذه القطاعات التي تمثل نطاقا واسعا من الطموحات والخفيات الاقتصادية والاجتماعية والثقافية. إن المسلمين الأمريكيين في وضع أفضل من غيرهم من المسلمين من حيث قدرقم على تنوير العالم الإسلامي عن مدة العولمة ومخاطرها، وتحل بذلك بعض المسائل المحورية التي تواجه الفكر الإسلامي المعاصر.

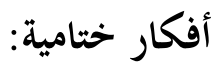

يتمحور العقل الغربي، الذي شكل أوضاع العالم منذ الثورة الصناعية، حول مفهوم التقدم، 70 ويبدو للبعض إن إمكانات التقدم غير محدودة، 71 فآفاق العالم الثالث في أبعادها المادية والعقلية كانت متاحة للاحتلال والاستعمار. ووفقا لأحد المؤلفين فإنه حسب الرؤية المعاصرة يضع معنى التقدم المرتبط بالخلاص- كاص كلا من التوسع الاقتصادي والإبداع التكنولوجي في بؤرة الاهتمام. 72 وفرضت ظاهرة التقدم تحديا رئيسيا على الثقافات التقليدية، وأسسها الاجتماعية الاقتصادية، ورؤاها الأخلاقية للعالم، ولم تتخل العولمة عن التقدم ولا حتى عن الأشكال الأشد تدميرا منه، كتلك التي تؤدي إلى شكل جديد من الاستعمار، فالمفهوم التقليدي للتقدم وفقا لرؤية حنا أرندت أدى إلى عملية لا تنتهي من مراكمة القوة الضرورية لحماية المراكمة التي لا تنتهي لرأس المال وهي التي حددت الأيديولوجية التقدمية لأواخر القرن التاسع عشر، وكذلك إيذانا

70

See Ibrahim M. Abu-Rabi', "Beyond the post-Modern". American Journal of Islamic Social Sciences 7 (September 1990): 235-56.

71 Jean-Marie Domenah, Enquete dur les ideas contemporaine (Paris: Points 1987).

Spretnak, Resurgence of the Real, 2. 
بصعود الإمبريالية. 73 إن العولمة تقودنا إلى نوعٍ جديد من الاستعمار أكثر تدميرا ودهاء من نظيره التقليدي، إضافة إلى ذلك خلقت العولمة - التجلي الأخير للعقل العدوائ الحديث- تشوشا حول ما هو المهم في الحياة. وتم إحلال مفاهيم جزئية جديدة لما تعنيه الحياة محل المفاهيم التقليدية للترابط والرخاء وبناء المجتمع. كانت نخبة الفكر الدينية في العالم الإسلامي، قبل بزوغ القرن العشرين، تعتبر التقدم الأوربي مجردا من أي أساس أخلاقي، ومع ذلك حاجج البعض بأن الغرب كان لديه العزم على مدى قرون طويلة على بناء أسس أخلاقية لا تتفق مع أسس الوحي التوحيدي.

وشجعت أخلاقيات العقل الغربي في ظل العولمة التراكم السريع للثورة وما يصحبها من قوة. فالنزعة الاستهلاكية الباذخة هي القاعدة، وفي رأي رتشارد فولك فإن تداعيات عولمة النفوذ الثقافي الغربي حاليا المتضمنة التزامها بالتحديث هي التي تسببت في أزمة النظام العالمي المتعددة الأبعاد من نزعات التسلح النووي والتصنيع والمادية واستهلاك.74

إنه لامفر من توليد وعي عام لفهم ومقاومة العوامل السلبية للعولمة ولمواجهة تداعياتما الهائلة. ويجب على العالم الإسلامي أن يكد لإحياء أخلاقيات الإسلام الاجتماعية والمالية والاقتصادية كظاهرة توحيدية لمقاومة هذه الانحرافات الخطيرة. ومن المهم إحياء مفهوم المجتمع الذي يقاوم هجمات النزعة الفردية التي أصبحت القاعدة اليوم في المجتمعات الصناعية المتقدمة وتوابعها في العالم الثالث، ومع تزايد الفجوة بين الشمال والجنوب، والفجوات بين الريف والحضر داخل معظم أقطار الجنوب، ومع العدد المتزايد من المهمشين والفقراء في المدن لا مفر م إحياء الخلق الإسلامي الجماعي الذي "يأمر بالمعروف وينهى عن المنكر". 75

Hannah Arendt, The Origins of Totalitarianism (Cleveland: Meridian Books, 1963), 143. 74 Richard Falk, Exploration at the Edge of Time: The Prospects for World Order (Philadelphia: Temple University Press, 1992), 48.

David R. Griffin and Huston Smith, Primordial Truth and Postmodern Theology (Albany: state University of New York Press, 1989). 
ومن المهم تذكر أنه لابد- لإبقاء رؤى العالم والفلسفات التقليدية حية في علام غلبت عليه الفردية

والتنافس القوي- من تقدم حلول على أساس رؤية تقليدية للعالمه ويجب أن تأخذ هذه الحلول في الحسبان الخراب النفسي والاجتماعي الذي أحدثنه العولمة في العالم الإسلامي، 76 بعبارة أخرى كيف يمكننا صياغة إجابات إسلامية على المشكلات الاقتصادية والثقافية والاختلالات التي خلقتها العولمة وحلفائها في العالم الإسلامي؟ ولا ننسى مثال أندونيسيا الملائم: هذا البلد الذي استهل برنامجا جسورا للتحديث مع اواخر الستينات واهمار اقتصاده فجأة في أوائل 1998. إن المرء ليتساءل بعجب عن الخطأ الذي جرى في هذا البلد! ومن المدهش أن تساوى ثروة الرئيس السابق سوهارتو وعائلته تقريبا القرض الذي وافق صندوق النقد الدولي على منحه اندونيسيا، لذا فإن السؤال هو: كيف نجحت النخب السياسية والعسكرية في العالم الإسلامي في جمع هذه الثروات غير المعقولة تحت حماية العولمة؟!

Hans Kung, A global Ethic for Global Politics and Economics (New York: Oxford University Press, 198).

76

Akbar S. Ahmed, Postmedernism and Islam: Perdicamentn and Promise (London: Routledge, 1992), Akbar S. Ahmed and Donna Hastings (eds). Islam, Globalization and postmodernity (London: Routledge,1994); Walden Bello, New Third World: Stategies for Survival in the Global Economy (London: Eathscan, 1990). 